\title{
23-Gauge Pars Plana Vitrectomy without Phacofragmentation for Dislocated Lens Fragments after Complicated Cataract Surgery
}

\author{
Gurkan Erdogan, ${ }^{1}$ Bugra Karasu, ${ }^{2}$ Abdullah Ozkaya, ${ }^{1}$ Irfan Perente, ${ }^{1}$ Zeynep Alkin, ${ }^{1}$ Muhittin Taskapili ${ }^{1}$ \\ 'Beyoglu Eye Training and Research Hospital, Istanbul, Turkey \\ ${ }^{2}$ Department of Ophthalmology, Pendik State Hospital, Istanbul, Turkey
}

\begin{abstract}
Objectives: The present study is an evaluation of outcomes of 23-gauge pars plana vitrectomy (PPV) performed to extract dislocated lens fragments without phacofragmentation after complicated cataract surgery.

Methods: In a consecutive case series, 50 eyes that underwent 23-gauge PPV without use of phacofragmatome were retrospectively reviewed.

Results: Visual acuity (VA) improved from preoperative $20 / 250$ to $20 / 57$ final VA $(p<0.0$ I). Mean surgical operating time for removal of lens material was 13.4 minutes (range: I-52 min). Mean length of time period between complicated cataract surgery and PPV was 8.4 days (range: $0-60 \mathrm{~d}$ ) and was negatively correlated with surgical time required $(P<0.0 \mathrm{l})$.

Conclusion: Comparable results were obtained with 23-gauge PPV performed without phacofragmentation for elimination of lens fragments following cataract surgery, making it a suitable alternative option. Amount of dislocated lens and the length of time since the cataract surgery are the main factors affecting operation time.

Keywords: Cataract surgery; dislocated lens fragment; 23-gauge vitrectomy.
\end{abstract}

\section{Introduction}

The surgical method employed to retrieve dislocated retained lens fragments after complicated cataract surgery is pars plana vitrectomy (PPV). Twenty-gauge PPV using a phacofragmatome is an efficient method for removal of lens fragments from the posterior segment (I-7). Recently, transconjunctival sutureless vitrectomy (TSV) using either 23- or 25-gauge blade has been reported to be successful in achieving same goal. In some instances of hard nuclei, sclerotomy site has been enlarged to 20 -gauge and phacofragmatome has been used, while in other reports, TSV has been performed without phacofragmatome (8-II). Although lens removal without phacofragmatome has the advantages of TSV, in cases of hard or large nuclei the process is prolonged. Choice of surgical technique depends on the subjective decision of the surgeon. In this study, results of 23-gauge PPV performed without phacofragmatome were evaluated as well as factors affecting surgical outcomes and feasibility.

\section{Methods}

This study is a retrospective, noncomparative, interventional case series. Total of 50 eyes of 50 consecutive patients with dislocated lens fragments following phacoemulsification who were treated with 23-gauge PPV at Umraniye Training and Research Hospital between May 2009 and June 2013 were

Address for correspondence: Gurkan Erdogan, MD. Umraniye Training and Research Hospital, Eye Clinic.

Kazım Karabekir Mah., Adem Yavuz Cad. No: 1, Umraniye, Istanbul, Turkey

Phone: +90 50535400 04, E-mail: erdogangurkan@yahoo.com

Submitted Date: October 19, 2016 Accepted Date: November 07, 2016 Available Online Date: November 15, 2016

${ }^{\circ}$ Copyright 2016 by Beyoglu Eye Training and Research Hospital - Available online at www.beyoglueye.com 
evaluated. Approval was obtained from the local ethics committee. Written informed consent was obtained from each patient and study followed the tenets of the Declaration of Helsinki. All patients had complete ophthalmic examination including Snellen best corrected visual acuity (BCVA), intraocular pressure (IOP) measurement with applanation tonometry, slit-lamp anterior segment and dilated fundus examination. B-scan ultrasonography was used to evaluate posterior segment in patients in whom fundus could not be seen ophthalmoscopically. Length of time between cataract surgery and PPV, quantity of lens material, duration of removal of material, and other details of PPV were recorded. Snellen VA score was converted to Logarithm of Minimum Angle of Resolution (logMAR) VA for statistical analysis. Patients with follow-up period of less than 2 months were excluded from the study.

All cases were operated on with standard 23-gauge vitrectomy techniques using Accurus 23-gauge Surgical Vitrectomy System (Alcon Laboratories Inc., Fort Worth, TX, USA) by a single surgeon. During surgery, non-contact wide-field viewing system (Optiflex, Volk Optical Inc., Mentor, OH, USA) was used to view the posterior segment. All patients received retrobulber anesthesia. Povidone iodine 10\% was applied around the eye contours and $5 \%$ solution was used in conjunctival sac. Corneal incisions from previous cataract surgery were examined and sutured when necessary. Three standard sclerotomy ports were created with 23-gauge transconjunctival trocar-cannula system. Following anterior segment intervention, posterior vitrectomy was performed and vitreous surrounding the lens material in the posterior segment was removed. Vitreous cutter and aspiration parameters were as follows: aspiration value was $400-450 \mathrm{mmHg}$ and vitreous cutter was at speed of 2000-2500 cuts per minute (CPM). Perflourocarbon liquid was used in some cases to protect macular region from damage. During stage when lens material was retrieved, vacuum pressure of cutter was increased to $600 \mathrm{mmHg}$ and cut rate was reduced to 800-1000 CPM. Backflushing through the tubing system was performed with an injector in case of obstruction of the vitrectomy probe. All of the patients were operated on without using phacofragmatome and without enlarging sclerotomy incisions.

In patients who had capsular support, foldable intraocular lenses (IOL) with polymethylmethacrylate haptics were placed in the ciliary sulcus. In patients without sufficient capsular support for sulcus fixation, IOL implantation was performed in a subsequent session. Subconjunctival gentamicin and dexamethasone injections were administered to all patients postoperatively. All of the patients were re-examined postoperatively on day I, day 3 , week I, first month, third month, and again every 6 months and monitored for complications.

\section{Statistical Analysis}

Number Cruncher Statistical System 2007 and Power Analysis and Sample Size 2008 software (NCSS, LLC, Kaysville, UT, USA) were used for statistical analysis. Descriptive statistics were evaluated. Preoperative and postoperative values were assessed using paired samples t-test for normally distributed parametric variables. Spearman's correlation analysis was used to evaluate parameters. $P$ values $<0.05$ were considered to be significant.

\section{Results}

Fifteen patients in the study were female and 35 were male. The average age was 70.6 years (range: $30-88$ y). Average follow-up period was 13.6 months (range, 2-48 months) (Table 1). None of the patients had glaucoma or uveitis in the operated eye. Average VA of the patients before PPV was determined to be 20/250 (logMAR acuity=1.09; range: hand motion at $2 \mathrm{ft}-20 / 28)$. Average IOP before surgery was $23.3 \pm 9.3 \mathrm{mmHg}$ (range: $9-46 \mathrm{mmHg}$ ).

Operations took place between 0 and 60 days (mean: 8.4 days) following cataract surgery.

Time period between cataract surgery and PPV was negatively correlated with surgical time for removal of lens material $(p<0.01)$. PPV was performed in 9 patients during the same session. Lens material left in the posterior segment varied from cortex to entire crystalline lens. This determination was made observationally by the surgeon during PPV. Ratio of retained lens in the posterior segment was as follows: In 10 cases, entire lens; in 6 cases, $75 \%$ of lens; in 10 cases, $50 \%$ of lens remained; in 7 cases, $33 \%$ of lens; in 8 cases, $25 \%$ of lens; and in 9 cases, there was only cortex material. Removal of lens material from within the eye took an average of 13.4 minutes (range: I-52 min). Significant correlation between quantity of retained lens and time required for removal of lens material was found $(p<0.05)$.

Eleven patients $(22 \%)$ had IOL implanted during cataract surgery. Secondary IOL implantation was performed on 23 patients (46\%) during PPV. Secondary IOL implantation was achieved in II patients (22\%) in a separate session after PPV.

Table I. Baseline characteristics and ophthalmic data
Number of eyes

Age, years (range)

Gender (female/male)

Time of IOL implantation

During cataract surgery

During PPV

Follow up, months (range)

IOL: intraocular lens; PPV: pars plana vitrectomy.

\section{0}

$70.6(30-88)$

$15 / 35$

II

39

$13.6(2-48)$ 
Three patients with degenerative myopia and I patient with cloudy cornea were followed up in aphakic state (8\%). One patient had localized retinal detachment during PPV. One patient had total retinal detachment in postoperative second month. These patients were successfully treated. Vitreous hemorrhage observed in 2 patients on first postoperative day cleared spontaneously. Submacular hemorrhage developed in I patient with wet-type age-related macular degeneration in the first postoperative week. In 6 cases, optical coherence tomography (OCT) revealed cystoid macular edema. Epiretinal membrane was detected in OCT in 2 patients in the final examination. Three patients needed anti-glaucoma medication in the postoperative period $(6 \%)$.

Mean postoperative IOP was $15.5 \pm 4.9 \mathrm{mmHg}$ on first day after PPV procedure and there was significant improvement compared to preoperative values $(p<0.01)$. Postoperative hypotony did not develop in any of the patients. On last postoperative examination, mean VA was 20/57 (logMAR acuity=0.4). There was significant improvement in VA postoperatively when compared with preoperative values $(p<0.01)$. None of the patients had endophthalmitis in the postoperative period.

\section{Discussion}

TSV has been increasingly performed since it was first introduced, and can be used for almost all cases where 20-gauge PPV was indicated, as well as management of dislocated lens fragments developing after surgery. Lens fragments can be removed with 23- or 25-gauge cutters. In cases of hard or large nuclei, 23- and 25-gauge ports can be enlarged to 20-gauge and retained material can be removed with phacofragmatome. In 23- and 25-gauge vitrectomy studies, removal of fragments without use of phacofragmatome was reported between $50 \%$ and $100 \%(8-1 \mathrm{I})$. In this study, procedures for all 50 patients, including patients with hard nuclei, were completed with 23-gauge cutter without phacofragmatome.

In their case series on 25-gauge vitrectomy, Kiss and Vavvas (8) reported that they enlarged I sclerotomy to 20-gauge in 3 of 6 cases (50\%). In another study, Ho et al. (9) reported that they managed 17 cases $(100 \%), 13$ of which were nuclear material, with 25 -gauge vitrectomy and without using phacofragmatome. They reported that average surgery time was 49 minutes (range: 18-96 min). Baker et al. (10) reported that they managed 12 of 20 cases $(60 \%)$ using only 23 -gauge vitrectomy system, while phacofragmatome was necessary in 8 cases. Horozoğlu et al. (I I) reported that they managed I5 of 17 cases ( $88 \%$ ) using only 23 -gauge vitrectomy system and phacofragmatome in 2 cases.

Previous studies have reported that patients treated with 20 -gauge vitrectomy had VA $\geq 20 / 40$ postoperatively in $44 \%$ to $72 \%$ of cases and II \% to $20 \%$ of the patients had postoperative VA equal to or less than 20/200 (I-7). In studies of TSV, $45 \%$ to $70 \%$ of patients had postoperative VA greater than $20 / 40$ and only $6 \%$ to $33 \%$ of patients had postoperative VA of less than 20/200 (8-II). In the present study, $42 \%$ of the patients had postoperative VA $\geq 20 / 40$ and $19 \%$ had postoperative VA $\leq 20 / 200$. This finding is consistent with previous studies.

In previous studies using 20-gauge technique, rates of postoperative complications were as follows: glaucoma: $2 \%-21 \%$, retinal detachment: $4 \%-21 \%$, and cystoid macular edema: $27 \%-28 \%(2-6)$. In studies using 23 - and 25 -gauge, reported postoperative complications were: glaucoma $0 \%-$ $5.9 \%$, retinal detachment: $0 \%-5 \%$, and cystoid macular edema: $0 \%-29.4 \%(8-11)$. In this study, we observed the following rates of these complications: glaucoma: $6 \%$, retinal detachment: $2 \%$, and cystoid macular edema: $16 \%$. Postoperative endophthalmitis was not seen in any of the cases. Our results are consistent with other studies in terms of postoperative complications.

In this study, 50 patients were treated with 23-gauge PPV without phacofragmatome and we found that average surgical time for removal of lens material from the vitreous was 13.4 minutes (range: I-52 min). There was a significant correlation between quantity of retained lens and time needed for removal of lens material $(p<0.05)$. Length of time between cataract surgery and PPV was negatively and significantly correlated with surgical time for removal of lens fragments $(p<0.05)$. Lens fragments in vitreous cavity are known to disintegrate with time. When we take this into consideration, it explains why lens material retained longer is more easily removed, as it becomes softer and more brittle over time.

Twenty-three gauge TSV without phacofragmatome is an optional method for management of dislocated lens fragments after complicated cataract surgery. In addition to various advantages of TSV, surgical difficulties in removing lens material with vitreous cutter and relatively longer duration may be seen as the disadvantages. There are no objective criteria for the choice of surgical technique and it remains the subjective decision of the surgeon. This study was designed to contribute to a better evaluation when making this decision. The lack of a control group is factor that limits this study. Further comparative studies are necessary to obtain a better understanding of transconjunctival PPV for dislocated lens fragments and to improve surgery.

\section{Disclosures}

Financial Disclosure: This retrospective study was not supported by any company. None of the authors has financial or proprietary interests in any material or method mentioned. This data has not been previously published. 
Peer-review: Externally peer-reviewed.

Conflict of Interest: None declared.

Authorship Contributions: Involved in design and conduct of the study (GE, BK, AO, IP, ZA, MT); preparation and review of the study (GE, AO, MT); data collection (GE, BK, IP); and statistical analysis (GE, ZA).

\section{References}

I. Greven CM, Piccione K. Delayed visual loss after pars plana vitrectomy for retained lens fragments. Retina 2004;24:363-7.

2. Margherio RR, Margherio AR, Pendergast SD, Williams GA, Garretson BR, Strong LE, et al. Vitrectomy for retained lens fragments after phacoemulsification. Ophthalmology 1997; 104: | 426-32. Crossret

3. Olsson RB, Ritland JS, Bjørnsson OM, Syrdalen P, Eide N, Overgård R. A retrospective study of patients with retained nuclear fragments after cataract extraction. Acta Ophthalmol Scand 2000;78:677-9, Crossre

4. Scott IU, Flynn HW Jr, Smiddy WE, Murray TG, Moore JK, Lemus DR, et al. Clinical features and outcomes of pars plana vitrectomy in patients with retained lens fragments. Ophthalmology 2003; I 10:1567-72. Crossre

5. Smiddy WE, Guererro JL, Pinto R, Feuer W. Retinal detach- ment rate after vitrectomy for retained lens material after phacoemulsification. Am J Ophthalmol 2003; I 35: I 83-7. Crossre

6. Ho LY, Doft BH, Wang L, Bunker $\mathrm{CH}$. Clinical predictors and outcomes of pars plana vitrectomy for retained lens material after cataract extraction. Am J Ophthalmol 2009;|47:587594 Crossre

7. Moore JK, Scott IU, Flynn HW Jr, Smiddy WE, Murray TG, Kim $\mathrm{JE}$, et al. Retinal detachment in eyes undergoing pars plana vitrectomy for removal of retained lens fragments. Ophthalmology 2003; I 10:709-14. Crossre

8. Kiss S, Vavvas D. 25-gauge transconjunctival sutureless pars plana vitrectomy for the removal of retained lens fragments and intraocular foreign bodies. Retina 2008;28:|346-51. Crossre

9. Ho LY, Walsh MK, Hassan TS. 25-Gauge pars plana vitrectomy for retained lens fragments. Retina 2010;30:843-9. Crossre

10. Baker PS, Spirn MJ, Chiang A, Regillo CD, Ho AC, Vander JF, et al. 23-Gauge transconjunctival pars plana vitrectomy for removal of retained lens fragments. Am J Ophthalmol 20I I;152:6247. Crossre

II. Horozoglu F, Yanyali A, Macin A, Nohutcu AF, Keskinbora $\mathrm{KH}$. 23-Gauge transconjunctival sutureless vitrectomy for retained lens fragments after complicated cataract surgery. Retina 2012;32:493-8. Crossre 